\title{
Cutaneous manifestations in a patient with chronic lymphocytic leukemia involving the head, neck and distal extremities
}

\author{
CHONGRONG LU ${ }^{1}$, LI LI ${ }^{1}$, QIAOHUA QIAO ${ }^{1}$, GUOZHEN LIU ${ }^{2}$ and LIZHENG FANG ${ }^{1}$ \\ ${ }^{1}$ Department of Family Medicine, Sir Run Run Shaw Hospital, School of Medicine, Zhejiang University, \\ Hangzhou, Zhejiang 310016, P.R. China; ${ }^{2}$ Family Medicine Residency Program, \\ Genesys Regional Medical Center, Grand Blanc, MI 48439, USA
}

Received May 9, 2014; Accepted December 24, 2014

DOI: $10.3892 /$ etm.2015.2178

\begin{abstract}
Chronic lymphocytic leukemia (CLL) infiltrating the skin is uncommon and can present in many different ways. The present study reports a case of CLL infiltrating multiple body areas. A 57-year-old male with a 10-year history of subclinical B-cell chronic lymphocytic leukemia (B-CLL) presented with skin hypertrophic changes of the ears, eyebrows, tip of the nose, toes and fingers. In addition, the patient had erythematous plaques on the buttocks. Histopathology revealed a lymphocytic infiltrate. The patient rejected the recommended chemotherapy and, following a three-year follow-up, remained alive with mildly aggravated symptoms. It has previously been reported that infiltrative CLL can involve the head and neck; however, involvement of multiple body areas, particularly toes and fingers is rare. This case highlights the importance of considering leukemia cutis in patients with underlying CLL who present with unusual clinical features.
\end{abstract}

\section{Introduction}

B-cell chronic lymphocytic leukemia (B-CLL) is the most common form of leukemia and occurs with a male predominance (1), with majority of patients being over the age of 45 years. Medical literature describing the appearance of cutaneous involvement in patients with B-CLL is limited. In the majority of cases, the cutaneous lesions are nonspecific manifestations associated with an impaired immune system (2). The reported specific skin lesions include nodules, papules, infiltrates, plaques, ulcerations and exfoliative erythroderma (2-5), presenting predominantly in the head and neck areas. In the present study, a 57-year-old man with B-CLL who presented with plaque skin infiltrates affecting the limbs, buttocks and prominent parts of the face is described.

Correspondence to: Professor Lizheng Fang, Department of Family Medicine, Sir Run Run Shaw Hospital, School of Medicine, Zhejiang University, 3 Qingchun East Road, Jianggan, Hangzhou, Zhejiang 310016, P.R. China

E-mail: lizheng.f@163.com

Key words: chronic lymphocytic leukemia, infiltrative, skin

\section{Case report}

A 57-year-old male presented with a one-week history of non-pitting edema in the hands and feet in addition to erythematous skin on both buttocks. The patient had a 10-year history of untreated B-CLL and self-reported concurrent gradual hypertrophic changes of the ears, eyebrows, nose and toes. Informed consent was obtained from the patient.

Blood tests showed a percentage of blood lymphocytes of $65.4 \%$ (normal, 20-45\%), a lymphocyte count of $5.7 \times 10^{9} / 1$ (normal, 1.5-3 $\times 10^{9} / 1$ ) and no significant abnormalities in erythrocyte sedimentation rate, tumor markers and biochemistry. A 24-h electrocardiogram showed atrial flutter and atrial fibrillation. The patient was admitted to Sir Run Run Shaw Hospital, School of Medicine, Zhejiang University (Hangzhou, China) for further diagnosis and treatment.

On examination, the vital signs were stable. The patient was observed to have plum-colored swelling involving the prominent parts of the ears (helix, tragus and ear lobe), the eyebrows, nose and toes and non-pitting edema on the dorsal surfaces of the hands and fingers (Fig. 1). The lymph nodes in the right submandibular, left subclavian, left axilla and groin areas were enlarged. Non-blanching erythematous plaques were present on the patient's buttocks.

Laboratory findings when the patient was first admitted were as follows: The leukocyte count was $14.0 \times 10^{9} / 1$ (normal range, $\left.4.0-10.0 \times 10^{9} / 1\right)$, the lymphocyte count was $10.6 \times 10^{9} / 1$ (normal range, $1.5-3.0 \times 10^{9} / 1$ ), the percentage of lymphocytes was $65.4 \%$ (normal range, 20-45\%), the neutrophil count was $2.9 \times 10^{9} / 1$ (normal range, $1.5-3.0 \times 10^{9} / 1$ ) and the percentage of neutrophils was $20.5 \%$ (normal range, $55-75 \%$ ). Histopathology of a biopsy from the right auricular lobule showed atypical hyperplasia of lymphoid tissue. Immunohistochemical investigation revealed that the right auricular lobule co-expressed CD20 and CD5, which is consistent with CLL/small lymphocytic lymphoma. Hematoxylin and eosin staining of bone marrow revealed the diffuse infiltration of small lymphocytic cells. Immunohistochemical staining revealed that the bone marrow was positive for the B-cell marker CD20 and also partly positive for CD23 and CD5, which is consistent with an infiltrate of CLL (Fig. 2).

The patient was recommended to receive chemotherapy but declined it due to a poor financial situation and fear of 

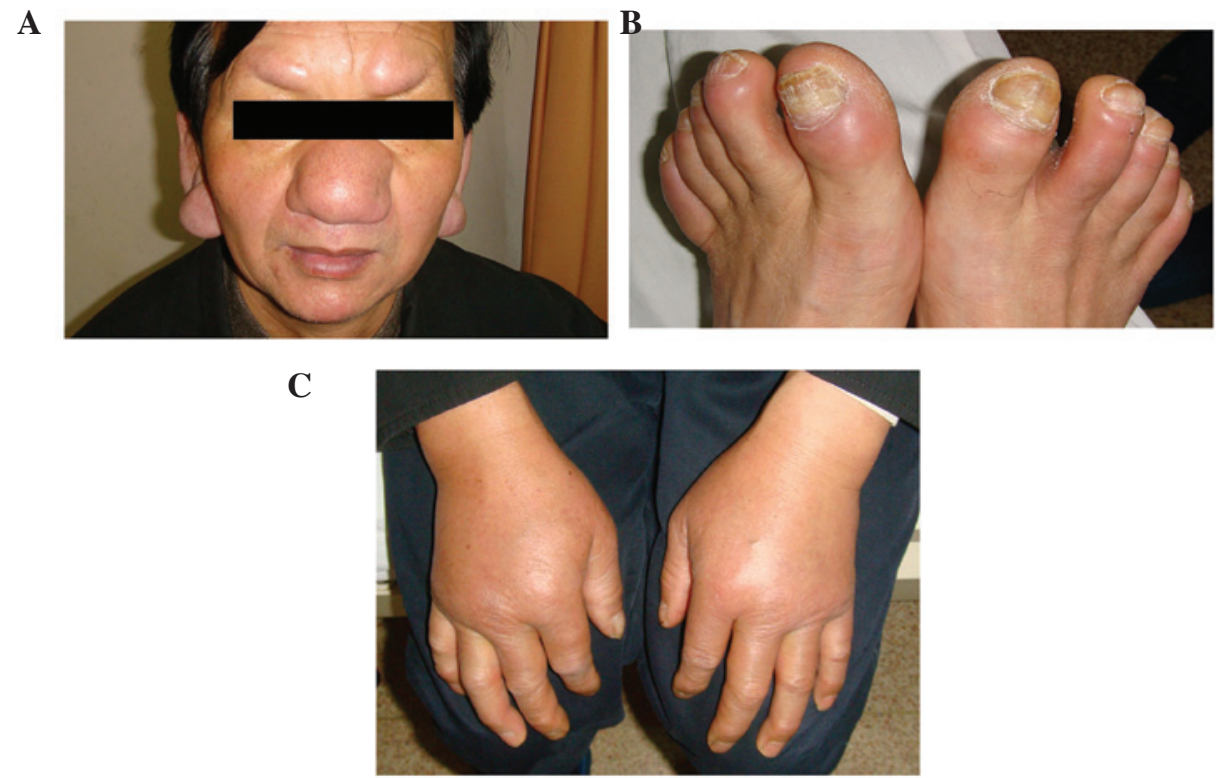

Figure 1. Appearance of the patient. Chronic lymphocytic leukemia infiltration of (A) the ears, eyebrows and nose and (B) the toes. (C) Non-pitting edema bilaterally on the back of the hands.
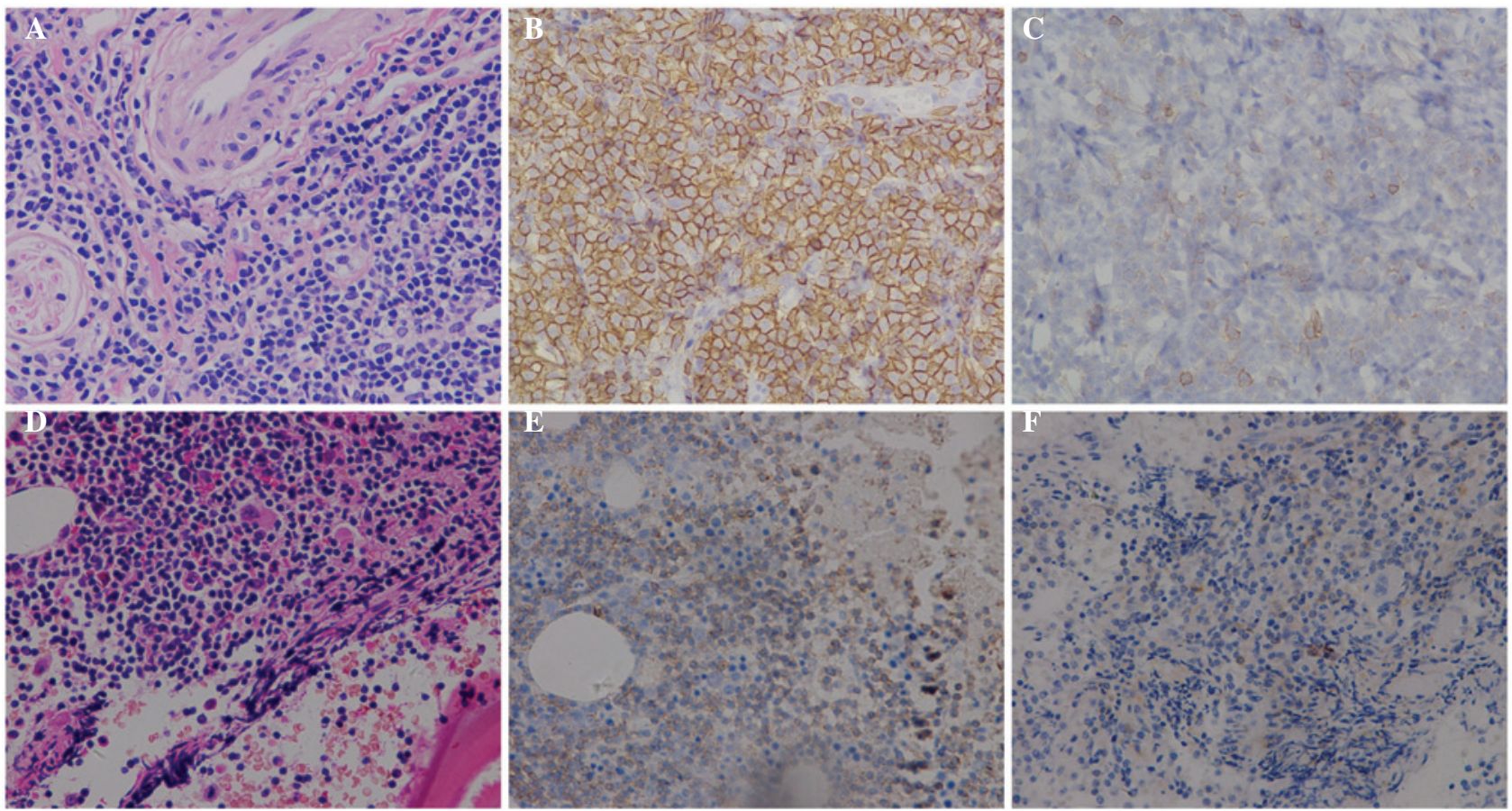

Figure 2. Biopsy results. (A) Histological examination of the right auricular lobule showed atypical hyperplasia of the lymphoid tissue (magnification, $\mathrm{x} 400$ ). Immunohistochemistry of the right auricular lobule revealed (B) $\mathrm{CD} 20^{+}$and $(\mathrm{C}) \mathrm{CD}^{+}$cells (magnification, $\mathrm{x} 400$ ). (D) Hematoxylin and eosin staining of bone marrow revealed the diffuse infiltration of small lymphocytic cells (magnification, $\mathrm{x} 400$ ). Immunohistochemistry of bone marrow revealed (E) $\mathrm{CD} 20^{+}$and (F) $\mathrm{CD} 23^{+}$cells (magnification, $\mathrm{x} 400$ ).

the side-effects of chemotherapy. Following a three-year follow-up in the clinic, the patient remained alive with mildly aggravated symptoms.

\section{Discussion}

B-CLL is a low-grade, B-cell lymphoproliferative monoclonal disorder in which functionally immunoincompetent lymphocytes are progressive accumulated, and thereby affect immune function and normal hematopoiesis. It is associated with an increased incidence of other malignancies, including squamous cell carcinoma, basal cell carcinoma, malignant melanoma and Merkel cell carcinoma (6). B-CLL patients are prone to cutaneous infections, particularly viral infections, and have exaggerated reactions to insect bites $(6,7)$.

From a broader point of view, the incidence of lymphomas, in particular that of B-CLL, is increasing worldwide $(8,9)$. The majority of patients with CLL manifest atypical clinical 
features. The most common symptoms and signs of this condition include fatigue, fever, easy bruising and generalized lymphadenopathy (10).

Although skin infiltration occurs in $3-50 \%$ of patients with leukemias or lymphomas overall, it is rare in patients with CLL $(11,12)$. When evident skin involvement is observed in CLL, it usually is seen in Richter syndrome or T-cell CLL $(13,14)$, which generally indicates a poor prognosis $(3,15,16)$. Medical literature reports that CLL can cause skin infiltrates, chronic and relapsing pruritic skin lesions (17) and indurated plaques of the eyebrows (18). Reports of cutaneous symptoms being the primary manifestation of B-CLL are unusual $(3,19,20)$. The case described in the present study presented as a skin infiltrate involving prominent parts of the head, such as the nose, ears and eyebrows, and other parts of the body, including the fingers and toes, a rare combination which might shed light on the mechanism involved in attracting leukemic cells of CLL patients to the skin. In the present case, this process was not investigated further and the possibilities can only be speculated on. The mechanism of cutaneous infiltration has not been fully elucidated. It has been postulated that skin invasiveness may be caused by the upregulation of intercellular adhesion molecule 1 (ICAM-1) and lymphocyte function-associated antigen 1 (LFA-1) (21). It is important to be aware of the possibility of these unusual presentations of cutaneous B-CLL, although skin involvement in B-CLL may be consistent with prolonged survival (15). Additional investigations into the behavior of B-CLL in the skin may further elucidate how this condition develops.

\section{References}

1. Chiorazzi N, Rai KR and Ferrarini M: Chronic lymphocytic leukemia. N Engl J Med 352: 804-815, 2005.

2. di Meo N, Stinco G and Trevisan G: Cutaneous B-cell chronic lymphocytic leukaemia resembling a granulomatous rosacea. Dermatol Online J 19: 20033, 2013.

3. Cerroni L, Zenahlik P, Höfler G, Kaddu S, Smolle J and Kerl H: Specific cutaneous infiltrates of B-cell chronic lymphocytic leukemia: a clinicopathologic and prognostic study of 42 patients. Am J Surg Pathol 20: 1000-1010, 1996.

4. Robak E and Robak T: Skin lesions in chronic lymphocytic leukemia. Leuk Lymphoma 48: 855-865, 2007.

5. Dhir R: Chronic lymphocytic leukaemia manifestating as exfoliative dermatitis. Indian J Dermatol Venereol Leprol 61: 102-103, 1995.

6. Agnew KL, Ruchlemer R, Catovsky D, Matutes E and Bunker CB: Cutaneous findings in chronic lymphocytic leukaemia. Br J Dermatol 150: 1129-1135, 2004
7. Walker P, Long D, James C and Marshman G: Exaggerated insect bite reaction exacerbated by a pyogenic infection in a patient with chronic lymphocytic leukaemia. Australas J Dermatol 48: 165-169, 2007.

8. Sng I: Malignant lymphoma - a changing spectrum. Ann Acad Med Singapore 38: 837-839, 2009.

9. Wu SJ, Huang SY, Lin CT, Lin YJ, Chang CJ and Tien HF: The incidence of chronic lymphocytic leukemia in Taiwan, 1986-2005: a distinct increasing trend with birth-cohort effect. Blood 116: 4430-4435, 2010.

10. Hallek M, Cheson BD, Catovsky D, et al; International Workshop on Chronic Lymphocytic Leukemia: Guidelines for the diagnosis and treatment of chronic lymphocytic leukemia: a report from the International Workshop on Chronic Lymphocytic Leukemia updating the National Cancer Institute-Working Group 1996 guidelines. Blood 111: 5446-5456, 2008.

11. Jasim ZF, Cooke N, Somerville JE and Hay RJ: Chronic lymphocytic leukaemia skin infiltrates affecting prominent parts of the face and the scalp. Br J Dermatol 154: 981-982, 2006

12. Chang HY, Wong KM, Bosenberg M, McKee PH and Haynes HA: Myelogenous leukemia cutis resembling stasis dermatitis. J Am Acad Dermatol 49: 128-129, 2003.

13. Giles FJ, O'Brien SM and Keating MJ: Chronic lymphocytic leukemia in (Richter's) transformation. Semin Oncol 25: 117-125, 1998.

14. Hoyer JD, Ross CW, Li CY, Witzig TE, Gascoyne RD, Dewald GW and Hanson CA: True T-cell chronic lymphocytic leukemia: a morphologic and immunophenotypic study of 25 cases. Blood 86: 1163-1169, 1995

15. Colburn DE, Welch MA and Giles FJ: Skin infiltration with chronic lymphocytic leukemia is consistent with a good prognosis. Hematology 7: 187-188, 2002.

16. Watson KM, Mufti G, Salisbury JR, du Vivier AW and Creamer D: Spectrum of clinical presentation, treatment and prognosis in a series of eight patients with leukaemia cutis. Clin Exp Dermatol 31: 218-221, 2006.

17. Neuber K, Berg-Drewniock B, Volkenandt M, et al: B-cell chronic lymphocytic leukemia associated with high serum IGE levels and pruriginous skin lesions: successful therapy with IFN-alpha 2 b after failure on IFN-gamma. Dermatology 192: $110-115,1996$.

18. Schmid-Wendtner MH, Sander C, Volkenandt $M$ and Wendtner CM: Unusual manifestations of B-cell disorders. Case 2: chronic lymphocytic leukemia presenting with cutaneous lesions. J Clin Oncol 17: 1084-1085, 1999.

19. Padgett JK, Parlette HL III and English JC III: A diagnosis of chronic lymphocytic leukemia prompted by cutaneous lymphocytic infiltrates present in Mohs micrographic surgery frozen sections. Dermatol Surg 29: 769-771, 2003.

20. Khandelwal A, Seilstad KH and Magro CM: Subclinical chronic lymphocytic leukaemia associated with a $13 q$ deletion presenting initially in the skin: apropos of a case. J Cutan Pathol 33: 256-259, 2006.

21. Uccini S, Ruco LP, Monardo F, La Parola IL, Cerimele D and Baroni CD: Molecular mechanisms involved in intraepithelial lymphocyte migration: a comparative study in skin and tonsil. J Pathol 169: 413-419, 1993. 